\title{
Venlafaxin ist kein SSRI, sondern ein SNRI, und das ist relevant
}

Jürgen Fritze ${ }^{1,2}$, Barbara Schneider2 ${ }^{2}$ Bernhard Weber ${ }^{2}$

${ }^{1}$ Verband der privaten Krankenversicherung, Köln

2Zentrum der Psychiatrie, Universität Frankfurt am Main

psychoneuro 2003; 29 (5): 240-244

Venlafaxin ist ein selektives Antidepressivum, indem es - wie selektiv-serotonerge Antidepressiva (SSRI) - frei von den anticholinergen, antihistaminergen, antiadrenergen und kardiotoxischen Nebenwirkungen der trizyklischen Antidepressiva ist. Venlafaxin hemmt aber - anders als die SSRI - nicht nur die synaptische Wiederaufnahme von Serotonin, sondern insbesondere in höherer Dosis auch die von Noradrenalin (sog. duales Wirkprinzip der selektiven SerotoninNoradrenalin-Reuptake-Inhibition, SNRI). Gemäß mehrerer Meta-Analysen ist die Rate von Vollremissionen bei Depression unter Venlafaxin signifikant höher als unter SSRI. Dieser Überlegenheit liegt wahrscheinlich der duale Mechanismus zu Grunde. Venlafaxin ist als einziges Antidepressivum auch für die Indikation der Rückfall- und Rezidivprophylaxe formal zugelassen. Ob das duale Wirkprinzip auch hier Vorteile bietet, müsste durch Vergleichsstudien geprüft werden. Venlafaxin ist neben Paroxetin das einzige zur Behandlung der generalisierten Angststörung formal zugelassene Antidepressivum. Ob das duale Wirkprinzip in dieser Indikation Vorteile bietet, müsste auch hier durch Vergleichsstudien geprüft werden.

\footnotetext{
S
} eit Entdeckung der Antidepressiva und Klärung ihrer mutmaßlichen Wirkprinzipien in den 50er-Jahren des letzten Jahrhunderts ist klar geworden, dass sich ihre Wirksamkeit nicht auf Depressionen beschränkt, sondern sich auf zahlreiche andere Krankheiten, u.a. die Gruppe der Angstkrankheiten, erstreckt. Die ätiopathogenetische Bedeutung dieser anscheinend nicht krankheitsspezifischen Modulation der Neurotransmission wird bisher schlecht verstanden. Die Erweiterung des Indikationsspektrums stellt auch höhere Anforderungen an die Arzneimittelverträglichkeit und -sicherheit.
Beginnend Mitte der 80er-Jahre des letzten Jahrhunderts wurden eine Reihe selektiv-serotonerger Antidepressiva, die Serotonin-Wiederaufnahmehemmer (SSRI) zugelassen. Ihre Entwicklung basierte auf der Serotonin-Mangelhypothese der Depression. Sie verfolgte das Ziel, bei gleicher Wirksamkeit die unerwünschten Begleitwirkungen der traditionellen, tri- und tetrazyklischen, unselektiven Antidepressiva zu vermeiden. Dazu gehört insbesondere die Sedierung und Gewichtszunahme durch Histamin-Rezeptorblockade, Mundtrockenheit und Obstipation durch Blockade muskarinischer Acetylcholinrezeptoren, Kreis- laufdysregulation durch Blockade $\alpha$ adrenerger Rezeptoren, und die Modulation von Ionenkanälen mit resultierender Kardiotoxizität insbesondere bei Überdosierung.

Die Entwicklung von Venlafaxin (Trevilor ${ }^{\circledR}$ retard) folgte dem Konzept der Selektivität, aber nicht der wohlgemerkt hypothetischen - Vorstellung, die serotonerge Transmission wäre in der Pathogenese der Depression entscheidend, sondern bezog die Noradrenalin-Mangelhypothese der Depression ins pharmakodynamische Konzept ein. Venlafaxin hemmt also hochselektiv die synaptischen Transporter von Serotonin und Noradrenalin: das duale Wirkprinzip der Serotonin-NoradrenalinWiederaufnahmehemmung (SNRI). Entsprechend ist Venlafaxin in der anatomisch-therapeutisch-chemischen Klassifikation (ATC-System) der Weltgesundheitsorganisation ein eigener Kode (ATC Code: N06AX16) in einer Klasse „andere Antidepressiva“ (ATC-N06AX) zugewiesen worden in Abgrenzung zu den SSRI (ATCN06A). Stellt sich die Frage: Hat das einen mehr als formalen Sinn? Unterscheidet sich Venlafaxin von den anderen selektiven Antidepressiva in klinisch relevanter Weise? 


\section{Zulassungsstatus}

Venlafaxin ist zugelassen bei depressiven Episoden in der Behandlung von Patienten mit depressiven Erkrankungen, Depressionen mit begleitenden Angstzuständen, und in der Behandlung der generalisierten Angststörung. Venlafaxin ist damit neben Paroxetin - das einzige Antidepressivum, für das Wirksamkeitsbelege bei generalisierter Angststörung vorliegen, die die Zulassung durch die europäische Behörde (EMEA: European Medical Evaluation Agency) erlaubten. Das soll nicht bedeuten, andere Antidepressiva einschließlich der SSRI wären in dieser Indikation unwirksam; jedoch haben sich die entsprechenden Hersteller nicht die Mühe des Wirksamkeitsnachweises in dieser hochprävalenten und damit wichtigen Indikation gemacht. Venlafaxin konfrontiert in dieser Indikation also nicht mit dem immer brisanter werdenden Problem des sog. Off-Label-Use, d.h. Arzneimittel nicht außerhalb der zugelassenen Indikationen zu Lasten der gesetzlichen Krankenkassen verordnen zu dürfen.

Venlafaxin ist als bisher einziges Antidepressivum zur Erhaltungstherapie und Rezidivprophylaxe depressiver Erkrankungen zugelassen: Prävention eines Rückfalls (Relapse) nach Remission der depressiven Symptomatik bzw. Prävention des Wiederauftretens (Rezidiv) neuer depressiver Episoden (Recurrence). Das soll wiederum nicht bedeuten, andere Antidepressiva einschließlich der SSRI wären nicht rezidivprophylaktisch wirksam; jedoch haben sich die entsprechenden Hersteller trotz einer Reihe positiver Studien nicht die Mühe des abschließenden Wirksamkeitsnachweises gemacht. Angesichts der Tatsache, dass rund $60 \%$ der Kranken innerhalb eines halben Jahres nach pharmakotherapeutisch induzierter Remission einer Depression einen sog. Rückfall (Relapse) und wiederum rund 60\% innerhalb von fünf Jahren ein Rezidiv (Recurrence) erleiden, kann die medizinische Bedeutung von Erhaltungstherapie und Rezidivprophylaxe kaum hoch genug eingeschätzt werden (4).

Auch hier ist zu befürchten, dass die gesetzlichen Krankenkassen die
Erhaltungstherapie und Rezidivprophylaxe mit anderen Antidepressiva als Venlafaxine als Off-Label-Use „entdecken“. Das könnte zumindest theoretisch bedeuten, dass mit den anderen Antidepressiva eine Therapie gemäß des Standes der Wissenschaft, der zumindest eine Erhaltungstherapie über sechs Monate nach Remission verlangt, unmöglich wäre. Venlafaxin konfrontiert mit diesem Problem nicht.

\section{Pharmakodynamik: Duales Wirkprinzip}

Venlafaxin und sein Hauptmetabolit O-Desmethylvenlafaxin (ODV) hemmen potent die synaptische Wiederaufnahme von Serotonin und Noradrenalin sowie schwächer auch Dopamin $(16,22)$. Die Inhibitionskonstante $\mathrm{K}_{\mathrm{I}}$ ist für Venlafaxin am Serotonin-Transporter $210 \mathrm{nmol}$ und am Noradrenalin-Transporter 640 nmol (zum Vergleich z.B. Citalopram 1,8 nmol bzw. $6100 \mathrm{nmol}$ ). Die Inhibitionskonstante $\mathrm{K}_{\mathrm{I}}$ ist diejenige Konzentration des Antidepressivums, die benötigt wird um in vitro 50\% der Transporter zu blockieren, also ein Maß für die Potenz, woraus sich die klinisch notwendige Dosis ableitet. Die Relationen der tierexperimentell in-vivo gemessenen $\mathrm{ED}_{50}$-Werte sind vergleichbar (30).

Da klinisch die antidepressive Wirkung nicht unmittelbar nach Applikation eines Antidepressivums eintritt, sondern sich allmählich über Tage bis Wochen entwickelt, müssen adaptive Veränderungen bedeutsam sein. Diese werden bisher unvollständig verstanden. Gemäß der sog. Beta-Down-Hypothese könnte die Herabregulation $\beta$ adrenerger Rezeptoren zu diesen adaptiven Veränderungen gehören. Tierexperimentell regulieren Venlafaxin und sein Hauptmetabolit ODV die Empfindlichkeit $\beta$-adrenerger Rezeptoren bereits nach einmaliger Applikation (und auch bei chronischer Gabe) herunter (21). Demgegenüber regulieren trizyklische Antidepressiva (TCA) die Empfindlichkeit $\beta$-adrenerger Rezeptoren nur nach chronischer Gabe herunter. Dasselbe gilt für die $\beta$-adrenerg stimulierbare cAMP-Synthese als second messenger. Diese präklini- schen Befunde weisen auf einen möglicherweise schnelleren Eintritt der klinisch-antidepressiven Wirkung unter Venlafaxin als unter TCAs hin.

Venlafaxin besitzt in-vitro keine relevante Affinität zu anderen Rezeptoren, sodass entsprechende Nebenwirkungen (z.B. anticholinerge, sedierende und kardiovaskuläre) nicht zu erwarten sind.

\section{- Pharmakokinetik}

Venlafaxin wird in der Leber über das Cytochrom $\mathrm{P}_{450}$-Isoenzym CYP2D6 metabolisiert. Hauptmetabolit ist O-Desmethylvenlafaxin (ODV), das vergleichbare pharmakodynamische Eigenschaften wie Venlafaxin besitzt; bei Komedikation mit Arzneimitteln, die CYP2D6 hemmen, kann die Konzentration der Muttersubstanz Venlafaxin ansteigen. Alle anderen Metaboliten sind pharmakologisch inaktiv. Die metabolische Elimination von Venlafaxin erfolgt außer über CYP2D6 auch über das Cytochrom $\mathrm{P}_{450}$-Isoenzym CYP3A4. Venlafaxin selbst hemmt CYP2D6 nur schwach. Die Isoenzyme CYP1A2, CYP2C9 und CYP3A4 hemmt Venlafaxin in-vitro nicht. Für CYP2D6, CYP1A2 und CYP3A4 wurde dies beim Menschen in Interaktionsstudien bestätigt. CYP2C19 wird offenbar ebenfalls nicht gehemmt, da Venlafaxin keine entsprechende Interaktion mit dem CYP2C19-Substrat Diazepam zeigt. (22).

\section{- Klinische Relevanz}

\section{Effektstärke bei Depressionen:}

In der Meta-Analyse von Smith et al. (31) wurden 32 doppelblinde, randomisierte Studien erfasst, die Venlafaxin mit anderen Antidepressiva verglichen. Hauptzielparameter waren die Unterschiede am Behandlungsende, ausgedrückt als standardisierte Effektstärke. Insgesamt war Venlafaxin signifikant wirksamer als die anderen Antidepressiva (standardisierte Effektstärke -0,14; 95 \% Konfidenzintervall $-0,007$ bis $-0,22$ ). In getrennten Vergleichen fand sich ein signifikanter Vorteil zu Gunsten Venlafaxin gegenüber SSRIs (20 Studien), nicht jedoch gegenüber TCAs (7 Studien). Danach teilt 
Venlafaxin also den Wirksamkeitsvorteil der TCA, ohne mit deren unerwünschten Begleitwirkungen belastet zu sein.

Demgegenüber fand die MetaAnalyse von Freemantle et al. (12) anhand von 107 Studien keine Unterschiede zwischen SSRI und dual wirkenden Antidepressiva. Diese Meta-Analyse prüfte kontinuierliche Outcome-Maße (z.B. HamiltonDepression-Score (HAM-D) oder Montgomery-Asberg-DepressionScore (MADRS)) und Responseraten. Sie ist kritisiert worden bezüglich der Validität der pharmakodynamischen Klassifikation der Antidepressiva im Vergleich zu SSRI (2).

Vollremission bei Depressionen:

Traditionell dominieren in den Zielvariablen von AntidepressivaStudien Mittelwertsvergleiche eines Depressions-Schweregrad-Maßes (z.B. Hamilton-Depression-Score (HAM-D) oder Montgomery-AsbergDepression-Score (MADRS)). Klinisch bedeutsamer sind aber die Vollremissionen, denn die Therapie kann sich nicht mit begrenzten Besserungen zufrieden geben, sondern muss auf Gesundung zielen.

Smith et al. (31) analysierten auch die Remissionsraten (HAM-D < 8). Venlafaxin hatte mit einer NNT (number needed to treat, d.h. Anzahl Patienten, die mit Venlafaxin behandelt werden müssen, um einem Patienten einen gegenüber den Vergleichssubstanzen zusätzlichen Therapieerfolg zu ermöglichen) von 14 einen signifikanten Vorteil (ODDSRatio 1,36, 95\% Konfidenzintervall 1,14-1,61) gegenüber den SSRI. Thase et al. (32) analysierten die Daten aus 8 methodisch vergleichbaren, randomisierten Doppelblindstudien zur Major Depression bezüglich der Vollremission (Hamilton-DepressionScore $(H A M-D) \leqq 7)$ unter Venlafaxin retard $(n=851)$ im Vergleich zu SSRI (Fluoxetin, Paroxetin, Fluvoxamin, $\mathrm{n}=748)$ bzw. Plazebo ( $\mathrm{n}=446 ; 4$ Studien). Die Remissionsrate unter Venlafaxin retard betrug $45 \%$, unter den SSRI 35\% und unter Plazebo 25\%. Die Unterschiede zugunsten Venlafaxin waren signifikant.

Die Meta-Analyse von Enstuah et al. (9) prüfte die Rate von Vollremis- sionen (HAM-D ${ }_{17} \leqq 7$ ) in 31 randomisierten, doppelblinden Studien mit 7031 Patienten (Venlafaxin: 3078, SSRIs (Fluoxetin, Sertralin, Paroxetin, Fluvoxamine, Citalopram): 3025 Plazebo: 928). Die Remissionsraten betrugen $41 \%$ für Venlafaxin, 34\% für die SSRIs und 24\% für Plazebo, wobei die Unterschiede zugunsten Venlafaxin sowohl gegenüber SSRIs als auch Plazebo statistisch signifikant $(p<0,001)$ waren. Die höhere Remissionsrate scheint dosisabhängig zu sein, d.h. bei höherer Dosis von Venlafaxin kommt zur serotonergen Wirkung die noradrenerge hinzu (24).

\section{Responserate bei Depressionen:}

Smith et al. (31) analysierten auch die Responseraten (Besserung um mindestens 50\%). Venlafaxin hatte mit einer NNT von 19 (ODDS-Ratio 1,27, 95\% Konfidenzintervall 1,07-1,52) einen signifikanten Vorteil gegenüber den SSRI. Die Meta-Analyse von Einarson et al. (7) prüfte die Responserate, definiert als Besserung des HAM-D- oder MADRS-Score um mindestens 50\%. Datenbasis war eine systematische Literaturrecherche, die 44 Studien mit 63 Studienarmen und 4033 Patienten mit Depressionen identifizierte. Hier zeigte Venlafaxin retard in 73,7\% der Fälle einen Erfolg der sich signifikant von den Erfolgsraten unter SSRIs $(61,1 \%)$ und unter TCAs $(57,9 \%)$ unterschied $(\mathrm{p}<0,001)$.

\section{Zeit bis zum Wirkungseintritt bei} Depression:

Unter Venlafaxin wurde wiederholt ein früher Wirkungseintritt beobachtet (5, 15, 25, 27-29). Mögliche Mechanismen für einen frühen Wirkungseintritt umfassen u.a. eine bei dualer Wirkung raschere Desensitivierung von $\beta$-adrenergen Rezeptoren: Auch die Kombination von Fluoxetin (SSRI) mit Desipramin (Noradrenalin-Aufnahmehemmer), quasi als Imitation des dualen Wirkprinzips von Venlafaxin, induziert eine schnellere Desensitivierung (3). Beitragen könnten auch die kurze Halbwertszeit von Venlafaxin sowie die kurze Zeitdauer bis zum Erreichen des Steady state und die Möglichkeit, retardiertes Venlafaxin rasch auf hohe Dosen zu erhöhen.
In einer Studie bei 358 ambulanten Patienten war eine statistisch signifikante therapeutische Wirkung von Venlafaxin in Woche 1 in der Gruppe mit hoher Dosierung und in Woche 2 mit mittlerer Dosierung zu Plazebo feststellbar (27). In einer weiteren Studie mit rascher Dosiseskalation auf $375 \mathrm{mg}$ in der ersten Woche zeigten sich statistisch signifikante Unterschiede ab Tag 4 (15). In der Studie von Salinas et al. (29) zeigte sich im paarweisen Vergleich eine signifikante $(p<0,05)$ Überlegenheit von Venlafaxin gegenüber Paroxetin in Woche 1, 2, 4, 6 und 8 , vereinbar mit einem früheren Wirkungseintritt. Ebenso zeigte die Meta-Analyse von Thase et al. (32) bereits ab der zweiten Woche eine signifikante $(p<0,05)$ Überlegenheit von Venlafaxin gegenüber SSRIs, ebenfalls vereinbar mit schnellerer Aufhellung der Depression. Dieser Effekt scheint dosisabhängig zu sein, d.h. bei höherer Dosis von Venlafaxin kommt zur serotonergen Wirkung die noradrenerge hinzu (24).

\section{Erhaltungstherapie und Rezidiv-} prophylaxe:

Die Wirksamkeit von Venlafaxin in der Erhaltungstherapie (relapse prevention) und Rezidivprophylaxe (recurrence prevention) der Depression wurde in doppelblinden, randomisierten Studien für bis zu zwölf Monate belegt ( 8 ) mit signifikanter $(p<0,019)$ Überlegenheit gegenüber Plazebo. In die Meta-Analyse von Entsuah et al. (8) zur Erhaltungstherapie (6 Monate) gingen vier Studien ein. Dabei war Venlafaxin mit einer Rückfallrate (Relapse) von 11\% (Plazebo 23\%) nominal auch dem Imipramin (13\%) und Trazodon (21\%) überlegen. Retardiertes Venlafaxin war dem nicht retardierten überlegen (33). In der Rezidivprophylaxe (12 Monate) beliefen sich die kumulativen Rezidivraten unter Venlafaxin auf $20 \%$, Imipramin $31 \%$, Trazodon 29\%, Plazebo 34\% (8). In einer jüngsten doppelblinden Rückfallpräventionsstudie (23), in der 235 $(n=213$ gingen in die ITT-Analyse ein) der über sechs Monate stabilen Venlafaxin-Responder (HAM-D ${ }_{21}$ $<10)$ doppelblind auf Plazebo $(n=107)$ randomisiert wurden oder 
auf Venlafaxin ( $n=106)$ blieben, blieb bei $67 \%$ unter Venlafaxin die Remission über zwölf Monate stabil gegenüber nur 46\% unter Plazebo $(p<0,01)$. Da nur Verrgleichsstudien gegen Imipramin und Trazodon vorliegen, könnten die Frage, ob auch hier das duale Wirkprinzip Vorteile bietet, nur direkte Vergleichstudien gegen andere selektive Antidepressiva beantworten.

\section{Wirksamkeit bei generalisierter} Angstkrankheit:

Da - abgesehen von Venlafaxin und Paroxetin - andere Antidepressiva bisher nicht für die Therapie der generalisierten Angststörung zugelassen sind, wurde die Wirksamkeit von Venlafaxin retard in dieser Indikation bisher nur gegen Plazebo über Behandlungszeiten bis zu 24 Monaten geprüft und belegt (1, 10 , 14, 17-19, 26). Demgegenüber liegen für Paroxetin nur Kurzzeitstudien (bis zu 8 Wochen) vor (15). Die Responseraten (Besserung um mindestens $50 \%$ ) nach sechsmonatiger Therapie liegen unter Venlafaxin bei 66\% gegenüber 39\% unter Plazebo, die Rate an Vollremissionen (Hamilton-Anxiety-Score (HAM-A) <8) bei $43 \%$ unter Venlafaxin gegenüber $19 \%$ unter Plazebo (18). Damit stellt Venlafaxin eine Referenzsubstanz für andere Pharmaka in dieser Indikation dar. Welchen spezifischen Beitrag gerade das duale Wirkprinzip von Venlafaxin hier leistet, lässt sich folglich derzeit nicht beantworten.

\section{Schlussfolgerung}

Das duale Wirkprinzip von Venlafaxin hat klinische Relevanz und grenzt Venlafaxin auch klinisch von anderen selektiven Antidepressiva ab. Noch unzureichend belegt ist, inwieweit hierfür seine selektiv-serotonergen Eigenschaften in unteren Dosierungen und seine zusätzlich selektiv-noradrenergen Eigenschaften in höheren Dosierungen verantwortlich sind. Während man die klinische Relevanz des beschleunigten antidepressiven Wirkungseintritts hinterfragen kann, so ist jedoch die höhere Rate von Besserungen und insbesondere Vollremissionen unter Venlafaxin auch unter Berücksichtigung der damit potenziell verbun- denen gesundheitsökonomischen Vorteile (z.B. seltenere Krankenhausbedürftigkeit und frühere Rückkehr an den Arbeitsplatz) zweifellos bedeutsam. Bisherige entscheidungsanalytische gesundheitsökonomische Modellrechnungen weisen darauf hin, dass sich die Überlegenheit von Venlafaxin tatsächlich auszahlt $(6,11)$. Die gesundheitsökonomischen Vorteile sollten in adäquaten Feldstudien überprüft werden.

\section{Summary}

Venlafaxine is a selective antidepressant in that - like the selective serotonin reuptake inhibitors (SSRI) - it is free from the anticholinergic, antihistaminergic, antiadrenergic and cardiotoxic side-effects of tricyclic antidepressants. Venlafaxine, however, differs from the SSRIs by inhibiting not only the synaptic reuptake of serotonin but - especially at higher dosages - also that of norepinephrine (the so-called dual mechanism of selective serotonin-norepinephrinereuptake-inhibition, SNRI). According to several meta-analyses the rate of full remissions of depression is significantly higher under venlafaxine than under SSRIs. This superiority is probably due to the dual mechanism of action. Venlafaxine is the only antidepressant formally approved for relapse and recurrence prevention. Further studies comparing venlafaxine to SSRIs are needed to test whether the dual mechanism is advantageous also in this indication. Venlafaxine is beside paroxetine the only antidepressant formally approved for generalized anxiety disorder. Again, further studies comparing venlafaxine to SSRIs are needed to test whether the dual mechanism is advantageous.

\section{Key Words:}

Venlafaxine - depression - efficacy dual mechanism of action - response rate

\section{Literatur}

1. Allgulander C, Hackett D, Salinas E. Venlafaxin extended release (ER) in the treatment of generalised anxiety disorder: twenty-four-week placebo-controlled doseranging study. $\mathrm{Br}$ J Psychiatry 2001; 179 : 15-22
2. Anderson IM. Meta-analytical studies on new antidepressants. Br Med Bull 2001; 57: $161-178$

3. Baron BM, Ogden A-M, Siegel BW. Rapid down regulation of $ß$-adrenoceptors by co-administration of desipramine and fluoxetine. Eur J Pharmacol 1988; 154: 125-134 4. Bauer M, Whybrow P, Angst J, Versiani M, Möller HJ, WFSBP Task Force on Treatment Guidelines for Unipolar Depressive Disorders. World Federation of Societies of Biological Psychiatry (WFSBP) Guidelines for Biological Treatment of Unipolar Depressive Disorders, Part 1: Acute and Continuation Treatment of Major Depressive Disorder. World J Biol Psychiatry 2002; 3: 5-43

5. Benkert $O$, Gründer $G$, Wenzel $H$, Hackett D. A randomized double-blind comparison of a rapidly escalating dose of venlafaxine and imipramine in inpatients with major depression and melancholia. J Psychiatr Res 1996; 30: 441-451

6. Doyle JJ, Casciano J, Arikian S, Tarride JE, Gonzalez MA, Casciano R. A multinational pharmacoeconomic evaluation of acute Major Depressive Disorder (MDD): A comparison of cost-effectiveness between venlafaxine, SSRIs and TCAs. Value in Health 2001; 4: $16-31$

7. Einarson TR, Arikian SR, Casciano J, Doyle JJ. Comparison of extended release venlafaxine, selective serotonin reuptake inhibitors, and tricyclic antidepressants in the treatment of depression: a meta-analysis of randomized controlled trials. Clin Ther 1999; 21:296-308

8. Entsuah AR, Rudolph RL, Hackett D, Miska S. Efficacy of venlafaxine and placebo during long-term treatment of depression: A pooled analysis of relapse rates. Int Clin Psychopharmacology 1996; 11: 137-145

9. Entsuah R, Huang H, Willard L. Venlafaxine and SSRIs: pooled remission analysis (Mega-analysis of 31 studies of $>7000$ patients). Poster bei: World Congress of Psychiatry (WCP) 2002 August 24-29; Yokohama, Japan.

10. Feighner JP, Entsuah AR, McPherson MK. Efficacy of once-daily venlafaxine extended release (XR) for symptoms of anxiety in depressed outpatients. J Affect Disord 1998; 47: 55-62

11. Freeman $\mathrm{H}$, Arikian S, Lenox-Smith A. Pharmacoeconomic analysis of antidepressants for major depressive disorder in the United Kingdom. PharmacoEconomics 2000; 18: 143-148

12. Freemantle N, Anderson IM, Young P. Predictive value of pharmacological activity for the relative efficacy of antidepressant drugs: Meta-regression analysis. $\mathrm{Br}$ J Psychiatry 2000; 177: 292-302

13. Gelenberg AJ, Lydiard RB, Rudolph RL, Aguiar L, Haskins JT, Salinas E. Efficacy of venlafaxine extended-release capsules in nondepressed outpatients with generalized anxiety disorder: A 6-month randomized controlled trial. JAMA 2000; 283: 3082-3088

14. Gorman JM. Treatment of generalized anxiety disorder. J Clin Psychiatry 2002; 63 (Suppl 8): 17-23

15. Guelfi JD, White C, Hackett D, Guichoux JY, Magni G. Effectiveness of venlafaxine in 
patients hospitalized for major depression and melancholia. J Clin Psychiatry 1995; 56: 450-458

16. Horst WD, Preskorn SH. Rev Contemporary Pharmacotherapy 1998; 9: 293-302

17. Katz IR, Reynolds CF 3rd, Alexopoulos GS, Hackett D. Venlafaxine ER as a treatment for generalized anxiety disorder in older adults: pooled analysis of five randomized placebo-controlled clinical trials. J Am Geriatr Soc 2002; 50: 18-25

18. Meoni P, Salinas E, Brault Y, Hackett D. Pattern of symptom improvement following treatment with venlafaxine $X R$ in patients with generalized anxiety disorder. J Clin Psychiatry 2001; 62: 888-893

19. Montgomery SA, Sheehan DV, Meoni P, Haudiquet V, Hackett D. Characterization of the longitudinal course of improvement in generalized anxiety disorder during long-term treatment with venlafaxine XR. J Psychiatr Res 2002; 36: 209-217

20. Moyer JA, Andree TH, Haskins JT. The preclinical pharmacological profile of venlafaxine: A novel antidepressant agent. (Abstr) Clin Neuropharmacol 1992; 15 (Suppl 1, part B): 435B 21. Moyer JA, Muth EA, Haskins JT. In vivo antidepressant profiles of the novel bicyclic compounds Wy-45,030 and Wy45,881,(Abstr) Soc Neurosci 1984; 10: 261

22. Muth EA, Haskins JT, Moyer JA, Husbands G, Nielson ST, Sigg E. Antidepressant biochemical profile of the novel bicyclic compound Wy-45,030, an ethyl cyclohexanol derivative. Biochem Pharmacol 1986; 35: 4493-4497

23. Nemeroff CB, Entsuah AR, Kunz N. Remission rates during long-term treatment of depression with venlafaxine, Abstractband der American Psychiatric Association, Philadelphia 2002 May 18-23

24. Redrobe JP, Bourin M, Colombel MC, Baker GB. Dose-dependent noradrenergic and serotonergic properties of venlafaxine in animal models indicative of antidepressant activity. Psychopharmacology 1998; 138: 1-8

25. Rickels K, Derivan A, Entsuah R Miska S, Rudolph R. Rapid onset of antidepressant activity with venlafaxine treatment. Depression 1995; 3: 146-153

26. Rickels K, Pollack MH, Sheehan DV, Haskins JT. Efficacy of extended-release venlafaxine in nondepressed outpatients with generalized anxiety disorder. Am J Psychiatry 2000;157 968-974

27. Rudolph RL, Entsuah AR, Aguiar L, Derivan A. Early onset of antidepressant activity of venlafaxine compared with placebo and fluoxetine in outpatients in a double-blind study. Abstract presented at the 11th annual meeting of the European College of Neuropschopharmacology 1998 Oct 31-Nov 4, Paris/France 28. Rudolph RL, Feiger AD. A double-blind, randomized, placebo-controlled trial of once-daily venlafaxine extended release (XR) and fluoxetine for the treatment of depression. Journal of Affective Disorders 1999; 56: 171-181

29. Salinas for the Venlafaxine Study Group. Once-daily extended release (XR) venlafaxine versus paroxetine in outpatients with major depression Abstract, Biolog. Psychiatry 1997; 42 : 1S-297S

30. Sanchez C, Hyttel J. Comparison of the effects of antidepressants and their metabolites on reuptake of biogenic amines and on receptor binding. Cell Mol Neurobiol 1999; 19: 467-89 31. Smith D, Dempster C, Glanville J, Freemantle N, Anderson I. Efficacy and tolerability of venlafaxine with selective serotonin reuptake inhibitors and other antidepressants: a meta-analysis. British Journal of Psychiatry 2002; 180: 396-494

32. Thase M, Entsuah A, Rudolph R. Remission rates during treatment with venlafaxine or selective serotonin reuptake inhibitors. British Journal of Psychiatry 2001; 178: 234-241

33. Wellington K, Perry CM. Venlafaxine extended-release: A review of its use in the management of major depression. CNS Drugs. 2001; 15: 643-669

Korrespondenzadresse:

Prof. Dr. med. Jürgen Fritze

Asternweg 65

50259 Pulheim
Georg Thieme Verlag

Rüdigerstraße 14

70469 Stuttga

Telefon (0711) 89 31-0

Telefax (0711) 8931-322

Verantwortliche Schriftleitung:

Markus Gastpar, Essen

Jürgen Fritze, Pulheim

Erweiterte Schriftleitung:

Günther Faust, Mainz

Manfred Fichter, Prien am Chiemse

Andreas Hufnagel, Essen

Joachim Morgner, Dresde

Götz-Erik Trott, Aschaffenburg

Wissenschaftlicher Beirat:

Thomas Becker, Leipzig; Horst Berzew

ski, Berlin; Lothar Blaha, Deggendorf;

Hans-Dieter Brenner, Bern; Pasquale

Calabrese, Bochum; Klemens Dieckhö-

fer, Bonn; Tilman Elliger, Köln; Volke

Faust, Ravensburg; Franz Joseph Freisleder, München; Michael Geyer, Leipzig;

Iver Hand, Hamburg; Gerhard Harrer,

Salzburg, Kurt Heinrich, Düsseldorf;

Paul Hoff, Aachen; Siegfried Kaumeier

Merzig; Roger Kirchner, Cottbus; Gerd

Merzig; Roger Kirchner, Cottbus; Gerd

Lechner, Graz; Walter E. Müller, Frank-

furt am Main; Dieter Naber, Hamburg;

Hartmut Radebold, Kassel; Helmut

Remschmidt, Marburg; Peter Riedere

Würzburg; Eckardt Rüther, Göttingen;

Jörn Peter Sieb, München; Wolfgang

Weig, Osnabrück; Wolfgang Werner,

Merzig; Roland Wörz, Bad Schönborn;

Manfred Wolfersdorf, Bayreuth

Bestellungen: Über den Buchhandel

oder direkt beim Verlag

Bezugsbedingungen:

Einzelheft $€ 9,-+$ Porto

lahresabonnement $€ 69$,-

inkl. MwSt. und Porto.

Für Medizinstudente

gegen Nachweis und Bankeinzug

$€ 45$,- inkl. MwSt. und Porto.

Ausland zuzüglich Versandkosten

(cash with order) $€ 15,80$ (Europa)

bzw. $€ 44,90$ (Airlift).

Der Abonnementpreis umfasst $12 \mathrm{Ka}$ lendermonate (Mindestlaufzeit). Abon nements laufen weiter, wenn nich 3 Monate vor Jahresende eine Abbestellung beim Verlag vorliegt.

Bei Nichtbelieferung im Falle höhere Gewalt, bei Störungen des Betriebsfriedens, Arbeitskampf (Streik, Aussperrung) bestehen keine Ansprüche gegen den Verlag.

Verlag und Copyright:

(C) 2003 by Georg Thieme Verlag,

Rüdigerstraße 14, 70469 Stuttgart.

Mit dem Abdruck des Beitrages erwirbt der Verlag das alleinige und ausschließliche Recht für die Veröffentlichung in sämtlichen Publikumsmedien sowi Übersetzungen in fremde Sprachen. Nachdruck, fotomechanische Wiedergabe und Speicherung in den Datenverarbeitungsanlagen, auch auszugsweise, nur nach schriftlicher Genehmigung des Verlages. Die Abbildungen in den Beiträgen stammen, wenn nicht anders vemerkt, von den jeweiligen Autoren. Fü Inhalt Vorschau und Titelseite werden Inhalt, Vorschau und Titelseite werde Abbildungen aus den beschriebenen $\mathrm{Ar}$ beiten verwendet, anderenfalls ist die
Herkunft ebenfalls im Inhaltsverzeichnis Herkunft ebe
deklariert.

Hinweis für Autoren:

Zur Veröffentlichung in der Zeitschrift anZur Originalarbeiten, Übersichtsarbeiten und Kasuistiken, die nicht auch gleichzeitig an anderer Stelle zur Veröffentlichung eingereicht sein dürfen. Richtlinien zur Abfassung des Manuskriptes können bei de Redaktion angefordert werden.

Die eingereichten Arbeiten für Editorial, Schwerpunkt/Brennpunkt und Übersicht/Originalarbeit werden einem pee review der Schriftleitung unterzogen. review der übrigen Rubriken und Der Inhalt der übrigen Rubriken und der Supplements
Redaktionsleitung:

Günther Buck

Telefon (0711) 89 31-4 40

Chefredaktion:

Katrin Wolf

Telefon (0711) 89 31-179

Katrin.Wolf@thieme.de

Redaktionsassistenz:

Sabine Bischoff

Telefon (0711) 8931-551

Telefax (0711) 8931-322

Internet-Adresse:

http://www.thieme.de

Verantwortlich für den Anzeigenteil:

pharmedia, Anzeigen-

und Verlagsservice GmbH

Anzeigenleitung:

Greta Weller

Telefon (0711) 8931-304

Greta.Weller@pharmedia.de

Zur Zeit gilt Anzeigenpreisliste

1.10.2002

Zeitschriftenvertrieb:

Telefon (0711) 8931-321

Herstellung:

Karl-Heinz Zobe

Telefon (0711) 89 31-441

Satz, Grafik, Layout:

Wolfgang Eckl, Andrea Stiller,

Karl-Heinz Zobe

Druck und Verarbeitung

W. Kohlhammer Druckerei GmbH + Co 70329 Stuttgart

Bankverbindung:

Deutsche Bank Stuttgart

Konto-Nr. 1420 017, BLZ 60070070

Landesbank Baden-Württemberg

Konto-Nr. 2055 723, BLZ 60050101

Postgiro Stuttgart

Konto-Nr. 45 000-705, BLZ 60010070

Erscheinungsweise: monatlich

Regularly listed in EMBASE

Hinweis:

Wie jede Wissenschaft ist die Medizin ständigen Entwicklungen unterworfen. Forschung und klinische Erfahrung erweitern unsere Erkenntnis, insbesondere was Behandlung, medikamentöse Therapie sowie Diagnostik (Laborwerte etc.) anbelangt. Soweit in dieser Zeitschrift Dosierungen, Applikationen oder Laborwerte erwähnt werden darf der Leser zwar darauf vertrauen, dass AutoLeser zwar daraufvert ren, Herausgeber und Verlag große Sorgfalt darauf verwandt haben, dass diese Angaben dem Wissensstand bei Fertigstellung entsprechen. Für Angaben über Dosierungsanweisungen, Applikationsformen und Laborwerte kann vom Verlag jedoch keine Gewähr übernommen werden. Jeder Benutzer ist angehalten, durch sorgfältige Prüfung der Beipackzettel der verwendeten Präparate und ggf. nach Konsultation eines Spezialisten festzustellen, ob die dort spezianter gegebene Empfehlung fur Dosierungen oderdie Beachtung von Kontraindikationen gegenüber der Angabe in dieser Zeitschrift abweicht. Eine solche Prüfung ist besonders wichtig bei selten verwendeten Präparaten oder solchen, die neu auf den Markt gebracht worden sind. Jede Dosierung oder Applikation erfolgt auf eigene Gefahr des Benutzers. Laborwerte müssen immer auf Ihre Plausibilität geprüft werden und sind abhängig vom jeweiligen Testgerät bzw. Testkit. Autoren und Verlat it. Autoren und Verlag appellieren an nauigkeiten dem Verlag mitzuteilen. 